\title{
Dose Dispenser Cartridge
}

National Cancer Institute

\section{Source}

National Cancer Institute. Dose Dispenser Cartridge. NCI Thesaurus. Code C149443.

Container intended for use in a dose dispenser, usually pre-filled with a medicinal product. 